\title{
Verification of quantitative requirements for GNSS-based railway applications
}

\author{
H. Mocek, A. Filip \& L. Bažant \\ Railway Infrastructure Administration, LIS, Czech Republic
}

\begin{abstract}
The objective of this paper is to verify whether requirements for GNSS-based railway telematic applications are met through GNSS Safety of Life (SoL) services. Measurement methodology was developed for this purpose. An analysis of the achievement of railway requirements was subsequently performed. This technique represents a contribution to the certification process of the GNSS system, which must prove that the required parameters are fulfilled. The analysis consists of: 1) evaluation of static measurements to verify GNSS system behaviour under standard conditions of GNSS Signal-In-Space (SIS) reception, and 2) analysis of dynamic tests focused on train position and protection level determination under variable conditions of GNSS SIS reception in a real railway environment. Experimental tests have been carried out using GPS/EGNOS receivers that meet requirements for the SoL service according to the RTCA DO-229D standard.
\end{abstract}

Keywords: GNSS, Signal-In-Space, certification, EGNOS, non-precision approach, Rayleigh distribution, overbounding, reliability, availability, SIRF III.

\section{Introduction}

Certification of European navigation satellite system Galileo must be carried out before this GNSS system can be used in railway applications, especially in safety-related ones. A contribution to the certification process was previously performed by determining the minimum quality requirements for GNSS-based railway applications (Mocek et al. [1]). The next step in the certification process should be the verification that the proposed requirements are fulfilled. This paper evaluates the fulfilment of railway requirements on the basis of experiments with GPS/EGNOS receivers that meet requirements for the SoL applications 
according to the RTCA DO-229D standard [2]. Significant improvements of SIS reception and availability of position determination are demonstrated by means of SIRF III technology.

\section{Railway requirements for GNSS}

While in non-safety related applications it is possible to consider the fulfilment of requirements through GNSS SoL services, such as EGNOS Precision Approach (PA), Non-Precision Approach (NPA), Galileo SoL Level A/ B, in safety railway applications it is obvious that the GNSS SoL service cannot meet the demanding requirements of railway safety and dependability. This is evident from: 1) requirements for the functional and technical safety included in railway safety standards, e.g. EN 50129, and 2) interpretation of the Galileo SoL quality measures in terms of RAMS (Filip et al. [3]). Therefore, the following text will deal with the analysis of requirements for non-safety related applications.

Requirements for four selected applications are summarized in table 1 . These requirements were derived on the basis of methodology developed in Mocek et al. [1]. From table 1 it is obvious that the first three applications have similar requirements for horizontal positioning accuracy, horizontal alert limit and maximum standard deviation, but different requirements for update time interval. The last application "Diagnostics of infrastructure" has completely different

Table 1: $\quad$ Requirements for GNSS-based railway applications.

\begin{tabular}{|c|c|c|c|c|}
\hline $\begin{array}{l}\text { Non-safety } \\
\text { railway } \\
\text { application }\end{array}$ & $\begin{array}{l}\text { Application 1: } \\
\text { Performance } \\
\text { charging of } \\
\text { railway } \\
\text { infrastructure }\end{array}$ & $\begin{array}{l}\text { Application 2: } \\
\text { Position } \\
\text { monitoring of } \\
\text { trains / wagons }\end{array}$ & $\begin{array}{c}\text { Application 3: } \\
\text { Fleet } \\
\text { management }\end{array}$ & $\begin{array}{l}\text { Application 4: } \\
\text { Diagnostics of } \\
\text { infrastructure }\end{array}$ \\
\hline $\mathrm{HA}_{\max }$ & $8.6 \mathrm{~m}$ & $10 \mathrm{~m}$ & $8.6 \mathrm{~m}$ & $5.7 \times 10^{-3} \mathrm{~m}$ \\
\hline HAL & $22 \mathrm{~m}$ & $25 \mathrm{~m}$ & $22 \mathrm{~m}$ & $0.01 \mathrm{~m}$ \\
\hline$\xi_{\max }$ & $3.5 \mathrm{~m}$ & $4 \mathrm{~m}$ & $3.5 \mathrm{~m}$ & $0.0035 \mathrm{~m}$ \\
\hline$P_{T P L, f f}$ & $1-3.8 \times 10^{-17}$ & $1-6.3 \times 10^{-22}$ & $1-3.8 \times 10^{-17}$ & $1-10^{-4}$ \\
\hline$K_{\min }$ & 8.7 & 9.9 & 8.7 & 4.291 \\
\hline$\Delta t$ & $10 \mathrm{~min}$ & $30 \mathrm{~s}$ & $5 \mathrm{~min}$ & $30 \mathrm{~min}$ \\
\hline$\lambda_{\max }$ & $4.17 \times 10^{-2} \mathrm{~h}^{-1}$ & $1.39 \times 10^{-2} \mathrm{~h}^{-1}$ & $1.39 \times 10^{-2} \mathrm{~h}^{-1}$ & $2.08 \times 10^{-2} \mathrm{~h}^{-1}$ \\
\hline $\mathrm{MTBF}_{\min }$ & $24 \mathrm{~h}$ & $72 \mathrm{~h}$ & $72 \mathrm{~h}$ & $48 \mathrm{~h}$ \\
\hline MTTR $_{\min }$ & $0.19 \mathrm{~h}$ & $0.25 \mathrm{~h}$ & $0.25 \mathrm{~h}$ & $0.22 \mathrm{~h}$ \\
\hline $\mathrm{MDT}_{\max }$ & $\begin{array}{c}70 \mathrm{~h}+(1- \\
\left.\mathrm{A}_{\mathrm{SIS}}\right) \mathrm{T}_{\mathrm{y}}\end{array}$ & $\begin{array}{c}30 \mathrm{~h}+(1- \\
\left.\mathrm{A}_{\mathrm{SIS}}\right) \mathrm{T}_{\mathrm{y}}\end{array}$ & $\begin{array}{c}30 \mathrm{~h}+(1- \\
\left.\mathrm{A}_{\mathrm{SIS}}\right) \mathrm{T}_{\mathrm{y}}\end{array}$ & $\begin{array}{c}39 \mathrm{~h}+(1- \\
\left.\mathrm{A}_{\mathrm{SIS}}\right) \mathrm{T}_{\mathrm{y}}\end{array}$ \\
\hline $\mathrm{MUT}_{\text {min }}$ & $\mathrm{A}_{\text {SIS }} \mathrm{T}_{\mathrm{y}}-70 \mathrm{~h}$ & $\mathrm{~A}_{\mathrm{SIS}} \mathrm{T}_{\mathrm{y}}-30 \mathrm{~h}$ & $\mathrm{~A}_{\text {SIS }} \mathrm{T}_{\mathrm{y}}-30 \mathrm{~h}$ & $\mathrm{~A}_{\text {SIS }} \mathrm{T}_{\mathrm{y}}-39 \mathrm{~h}$ \\
\hline $\mathrm{A}_{\min }$ & $\begin{array}{c}99.2 \%-(1- \\
\left.\mathrm{A}_{\mathrm{SIS}}\right) \\
\end{array}$ & $\begin{array}{c}99.7 \%-(1- \\
\left.A_{\text {SIS }}\right)\end{array}$ & $\begin{array}{c}99.7 \%-(1- \\
\left.A_{\text {SIS }}\right)\end{array}$ & $\begin{array}{c}99.6 \%-(1- \\
\left.A_{\text {SIS }}\right)\end{array}$ \\
\hline $\mathrm{P}_{\mathrm{fm}, \max }$ & $5.42 \times 10^{-7}$ year $^{-1}$ & $7.63 \times 10^{-9}$ year $^{-1}$ & $1.9 \times 10^{-7}$ year $^{-1}$ & $1.4 \times 10^{-4}$ year $^{-1}$ \\
\hline
\end{tabular}


requirements for these quality measures. Therefore, the analysis of the proposed requirements will be summarized together for the first three applications and performed separately for the last application.

With regard to various railway environments, the value of Horizontal Alert Limit (HAL) can be increased to 50-100 m for many railway applications, including safety-related ones. This should have a positive impact on the reliability of the GNSS position determination.

$\mathrm{HA}_{\max }$
$\mathrm{HAL}$
$\xi_{\max }$

$\Delta t$
$P_{\text {TPL,ff }}$
$K_{\min }$
$\lambda_{\max }$
$\mathrm{MTBF}_{\min }$
$\mathrm{MTTR}_{\min }$
$\mu_{\max }$
$\mathrm{A}_{\mathrm{SIS}}>1 \%$
$\mathrm{MDT}_{\max }$
$\mathrm{MUT}_{\min }$
$\mathrm{A}_{\min }$
$\mathrm{P}_{\mathrm{fm}, \max }$
$\mathrm{T}_{\mathrm{y}}=8760 \mathrm{~h}$

Horizontal Accuracy

Horizontal Alert Limit

Estimated standard deviation of the model cumulative

distribution function that overbounds the GNSS position

uncertainty along semi-major axis of the error ellipse in xy plane

Update time interval

Probability of correct position determination of the Train

Position Locator (TPL)

Confidence coefficient

Failure rate

Minimum value for required Mean Time Between Failure. In practice, the actual MTBF is much higher.

Mean Time to Repair

Repair rate

SIS availability on the track

Mean Down Time

Mean Up Time

Minimum service availability

Maximum probability of major failures

Time interval (1 year)

\section{Evaluation of static measurements}

Static measurements were performed in the laboratory at the known position of the GNSS antenna. The aim of these experiments was to evaluate the correctness of calculation of GNSS data that will be further used to verify the dependability requirements of railway applications. The determination of dependability from a railway user point of view means the evaluation of the reliability and availability of GNSS position determination. Since the attributes of dependability primarily depend on the accuracy of GNSS positioning and GNSS position error is unknown for the user, it will be necessary to deal with the evaluation of probability distribution of position error and find out whether this distribution satisfies the assumptions given in [4].

Fig. 1 shows the record of static measurement from the GPS/EGNOS receiver PolaRx3 in the SBAS (Space Based Augmentation System) En-route/ NPA mode. The duration of this measurement is $26375 \mathrm{~s}$ (7.3 hours). The elevation mask was set to 5 degrees and with a recording time interval of $1 \mathrm{~s}$. Fig. 1 shows the time dependence of the Horizontal Protection Level (HPL), the real 
Horizontal Position Error (HPE), the estimated maximum standard deviation $\xi_{\text {max }}$ and the number of received satellites (SV). The reference antenna was situated in a stationary point with good visibility to satellites. The minimum number of visible satellites was 6 and the average number of received satellites was 8.6.

During these tests, under very good SIS reception conditions, the values of horizontal positioning error HPE were less than $2.7 \mathrm{~m}$. For the calculation of GNSS integrity risk it is supposed [4] that the horizontal position error is chi-square distributed and the integrity risk in the horizontal plane is calculated on the basis of the Rayleigh distribution with parameter $\xi_{\text {max }}$. The GNSS receiver estimates the standard deviation $\xi_{\max }$ in each epoch of measurement (fig. 1). Values of $\xi_{\max }$ are used to calculate the horizontal protection level HPL with a probability of missed detection $P_{M D}=5 \times 10^{-9}$ and the corresponding coefficient $K_{M D}=6.18[2]$.

For a verification of assumptions in [4] it was necessary to carry out more detailed analysis of HPE probability distribution. The histogram of HPE is shown in the fig. 2(a) bar graph. In order to investigate the characteristics of the probability distribution of HPE, the position errors $x_{\text {error }}, y_{\text {error }}$ in orthogonal directions $\mathrm{x}, \mathrm{y}$ were tested for the hypothesis that both variables have Gaussian probability distributions. Based on the results of the Jarque-Bera test, Liliefors test, chi-square goodness-of-fit test and analysis of the curves of Q-Q plots, see fig. 2(b) and fig. 2(c), Gaussian distributions can be assumed for these errors with the following parameters: $x_{\text {error }} \sim \mathrm{N}\left(\mu_{x}=0.308 \mathrm{~m}, \sigma_{x}^{2}=0.08 \mathrm{~m}^{2}\right), y_{\text {error }} \sim$ $\mathrm{N}\left(\mu_{y}=0.91 \mathrm{~m}, \sigma_{y}^{2}=0.14 \mathrm{~m}^{2}\right)$. Then the resulting horizontal position error HPE has two-dimensional normal probability distribution with the probability density

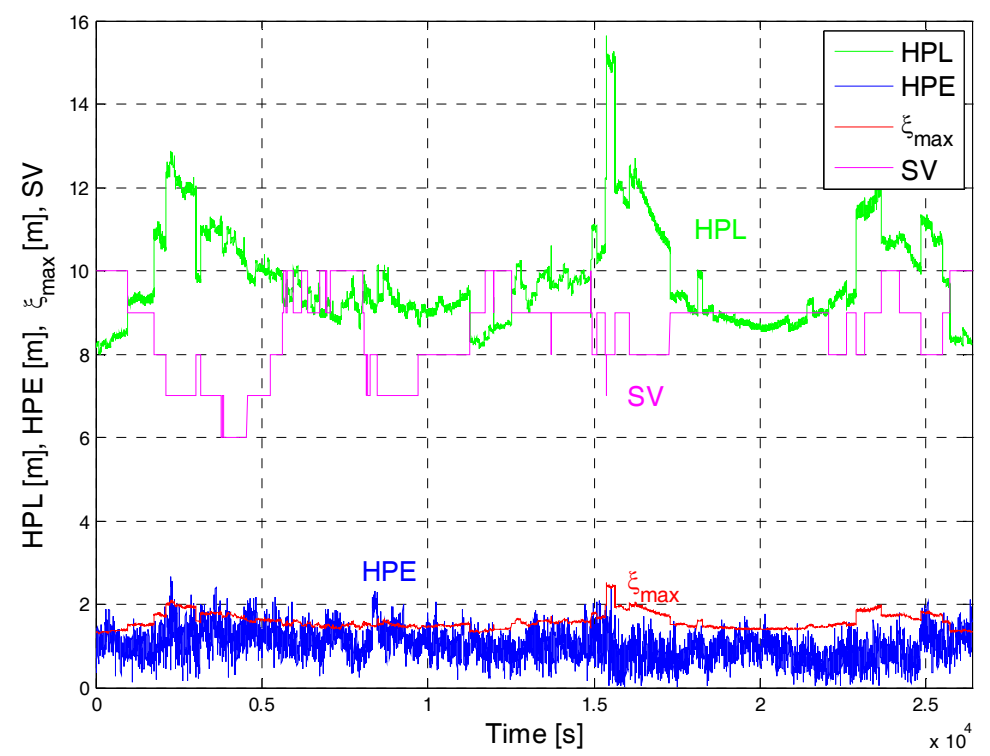

Figure 1: Time dependency of measured data from the receiver PolaRx3 in En-route/NPA mode. 


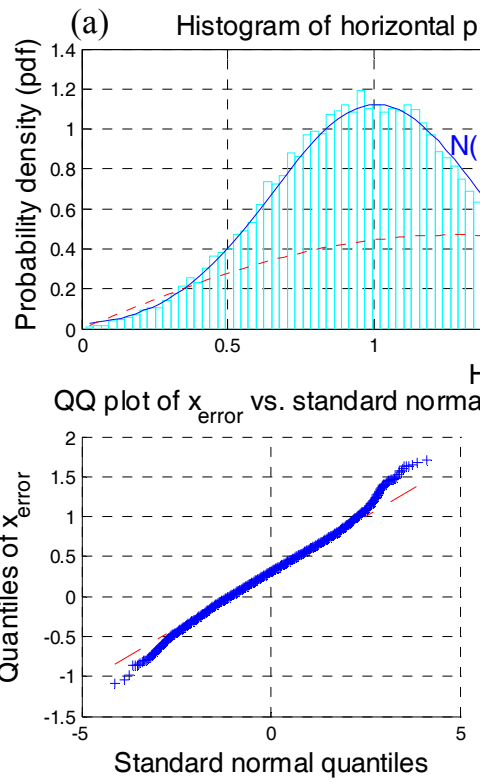

(b)

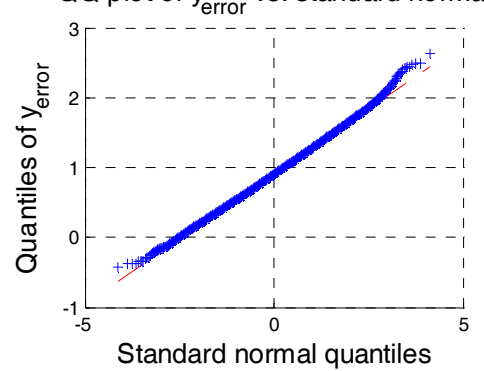

(c)

Figure 2: Histogram of horizontal position error and Q-Q plots of orthogonal position errors $x_{\text {error }}, y_{\text {error }}$ versus standard normal (colour online only).

$$
f(x, y)=\frac{1}{2 \pi \sigma_{x} \sigma_{y} \sqrt{1-\rho^{2}}} e^{-\frac{1}{2\left(1-\rho^{2}\right)}\left[\frac{\left(x-\mu_{x}\right)^{2}}{\sigma_{x}^{2}}-2 \rho \frac{\left(x-\mu_{x}\right)\left(y-\mu_{y}\right)}{\sigma_{x} \sigma_{y}}+\frac{\left(y-\mu_{y}\right)^{2}}{\sigma_{y}^{2}}\right]},
$$

where $\rho$ is correlation coefficient $\rho=\frac{\sigma_{x y}}{\sigma_{x} \sigma_{y}}$.

The probability that the HPE exceeds the horizontal alert limit HAL (the double integral cannot be expressed explicitly) corresponds to the failure of position determination (Mocek et al. [1]), which is considered as horizontal integrity risk in [4]:

$$
P\left(|H P E|>H A L \mid H_{0}\right)=1-P_{T P L, f f}=1-\iiint_{x^{2}+y^{2} \leq H A L^{2}} f(x, y) d x d y .
$$

The resulting error in the horizontal plane generally does not have the character of the Rayleigh distribution, since conditions for such distribution are not fulfilled: 1) errors in the orthogonal directions must be normally distributed, 2 ) they have to be independent, 3) they have zero mean values and 4) they have 
the same variances. From eqn. (2) it can be derived that the failure of GNSS positioning corresponds to the Rayleigh distribution only under these conditions:

$$
\begin{aligned}
P(|H P E|> & \left.H A L \mid H_{0}, \rho=0, \mu_{x}=\mu_{y}=0, \sigma_{x}=\sigma_{y}=\sigma\right)= \\
& =e^{-\frac{H A L^{2}}{2 \sigma^{2}}}=1-c d f_{\text {Rayleigh }}(H A L, \sigma)=P_{\text {fail,Rayleigh }} .
\end{aligned}
$$

However, not all of these conditions are fulfilled in the case of given static GNSS data. Two-dimensional normal distribution of HPE can be approximated by normal distribution instead of Rayleigh: $\operatorname{HPE} \sim \mathrm{N}\left(\mu_{H P E}=1 \mathrm{~m}\right.$, $\sigma_{H P E}^{2}=0.13 \mathrm{~m}^{2}$ ). The probability density of this normal distribution is depicted with the blue solid line in fig. 2(a). The red dashed line shows the probability density of the Rayleigh distribution with the smallest standard deviation $\min \left(\xi_{\max }\right)=1.3 \mathrm{~m}$. Normal distribution very well matches to the HPE, as is evident from figs. 2 and 3, and this was also demonstrated by the numerical integration of eqn. (2) for several values of HAL.

The probability distribution of the GNSS positioning error is supposed to be bounded by the Rayleigh distribution with parameter $\xi_{\max }$. Since the probability distribution of HPE is approaching the Gaussian distribution, the probability distribution of the position error has to overbound to this Gaussian distribution, as is illustrated in fig. 3 .

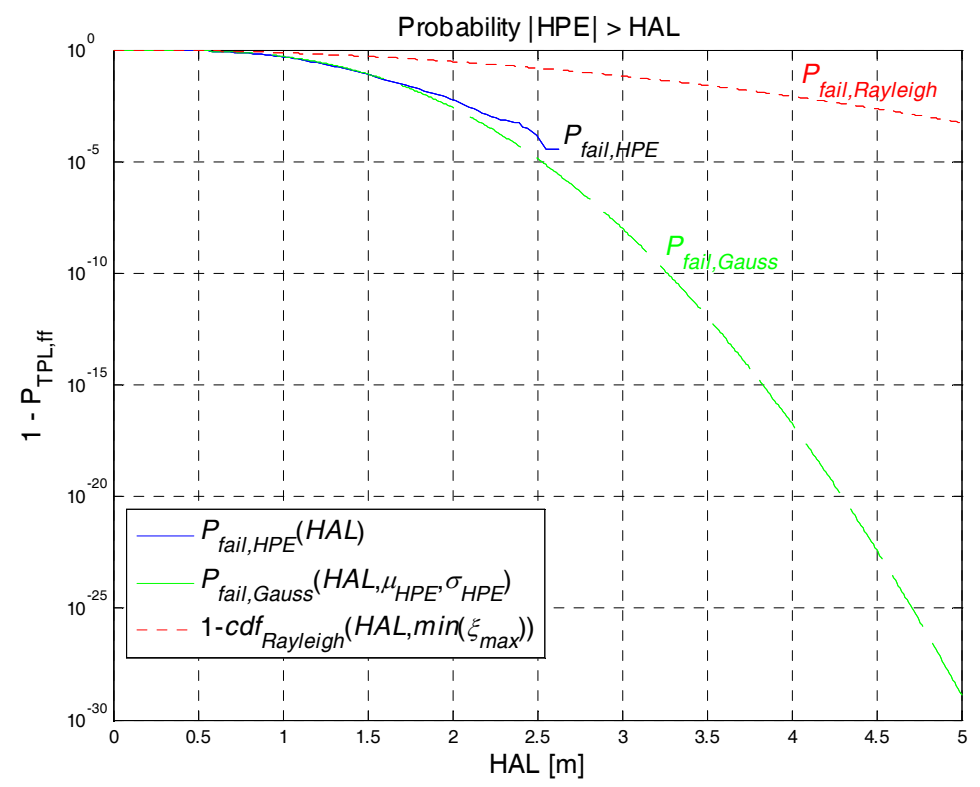

Figure 3: Failure probability of position determination: - static measurement, - - Gaussian distribution, - - - considered Rayleigh distribution from GNSS. 
By using inequality (4) it is possible to prove that the normal distribution is in this case bounded by the Rayleigh distribution, given that $\xi_{\max } \geq 0.87 \mathrm{~m}$ :

$$
\operatorname{erfc}(x) \leq \frac{2}{\sqrt{\pi}} \frac{e^{-x^{2}}}{x+\sqrt{x^{2}+\frac{4}{\pi}}}
$$

We want to prove that

$$
P_{\text {fail,Rayleigh }} \geq P_{\text {fail,Gauss }}\left(H A L, \mu_{H P E}, \sigma_{H P E}\right),
$$

where

$$
\begin{array}{r}
P_{\text {fail,Gauss }}\left(H A L, \mu_{H P E}, \sigma_{H P E}\right)=1-c d f_{\text {Gauss }}\left(H A L, \mu_{H P E}, \sigma_{H P E}\right) \\
+c d f_{\text {Gauss }}\left(-H A L, \mu_{H P E}, \sigma_{H P E}\right) \cong \frac{1}{2} \operatorname{erfc}\left(\frac{H A L-\mu_{H P E}}{\sqrt{2} \sigma_{H P E}}\right)
\end{array}
$$

Let's use a custom substitution of $x=\frac{H A L-\mu_{H P E}}{\sqrt{2} \sigma_{H P E}}$. After substitution of eqns. (3) and (6) to eqn. (5), and applying inequality (4) we get

$$
\xi_{\max } \geq \max \left\{\frac{\mu_{H P E}+\sqrt{2} \sigma_{H P E} x}{\sqrt{2\left[x^{2}+\ln \sqrt{\pi}+\ln \left(x+\sqrt{x^{2}+\frac{4}{\pi}}\right)\right]}}\right\} \approx 0.87 \mathrm{~m} .
$$

This overbounding is valid from the specific alert limit exceeding value of $H A L_{\min }$. The dependence between parameter $\xi_{\max }$ and $H A L_{\min }$ can be derived on the assumption that the probability $P_{\text {fail,Rayleigh }}$ is equal to the $P_{\text {fail,Gauss }}$. After using eqns. (3) and (6) we obtain

$$
\xi_{\max } \cong \frac{H A L_{\min }}{\sqrt{-2 \ln \left[\frac{1}{2} \operatorname{erfc}\left(\frac{H A L_{\min }-\mu_{H P E}}{\sqrt{2} \sigma_{H P E}}\right)\right]}} .
$$

$H A L_{\min }$ value cannot be explicitly expressed from eqn. (8). It must be determined by numerical iteration. For $\min \left(\xi_{\max }\right)=1.3 \mathrm{~m}$ the value of $H A L_{\min }=$ $0.46 \mathrm{~m}$ can be obtained. Since the minimum standard deviation also satisfies the condition given by eqn. (7), the probability of failure of GNSS positioning is 
always considered higher for horizontal alert limit greater than $H A L_{\min }$. Rayleigh distribution very well overbounds calculated Gaussian distribution, especially for higher values of HAL. This is also valid for all Rayleigh distributions supposed during the whole measurement.

After verification of behaviour of the GNSS system with regard to the positioning accuracy, we can proceed with the analysis of requirements for railway applications. For the first three applications, the maximum position error does not exceed the estimated maximum standard deviation $\xi_{\max }$. Horizontal alert limit in the range of $22 \mathrm{~m}$ to $25 \mathrm{~m}$ fits well with the use of the protection level of the EGNOS system with regard to the occurrence of major failures. The real position error does not exceed HAL or HPL. Reliability and availability of GNSS positioning for the given static measurement is $100 \%$.

Regarding the last application "Diagnostics of infrastructure" there is not possible due to its character to make a significant change of acceptable value of HAL. Horizontal accuracy of the EGNOS system was identified as 1.6 meters in $95 \%$ of cases. This value is much higher than desired HAL of $\mathrm{cm}$ level. Requirements for this application cannot be met by means of EGNOS system. Other GNSS systems or future Galileo system will be also unable to meet such stringent accuracy requirement, because the accuracy of SBAS differential systems is in the range of meters. Since the application does not require frequent sending of position information $(30 \mathrm{~min})$ and the probability of occurrence of major failures is much lower in comparison with the other applications, the solution for this application could potentially be the usage of the GNSS RTK mode (cm level accuracy) in combination with longer static measurements.

\section{Dynamic performance verification}

\subsection{Experiments carried out on the track}

Experimental dynamic verification with the GNSS receivers PolaRx3 in Enroute/ NPA mode was realized with a mobile robot on the test track Pardubice Nemošice. Another measurement was carried out with the measuring rail vehicle on the track Pardubice - Brno - Strelice. The purpose of these tests was to verify position and protection level determination with respect to dynamic behaviour of the GNSS receiver in real conditions on the track. Dependability attributes for each application have been also derived.

Static measurement described in the previous chapter was characterized by a very good SIS reception. Position and protection levels were continually computed and provided from the GNSS receiver. The situation is completely different for dynamic measurement under real railway conditions, when the GNSS position and protection level determination are influenced by different availability of SIS due to local obstacles along the track. GNSS receiver does not determine its position or protection level in some cases of partial or full SIS blocking. However, the greatest influences on the positioning have the transition states characterized by intermittent reception of SIS, see fig. 4. HPL values can 
then reach several tens of meters. This reduces the possibility of using such information. Nevertheless, if continuous SIS reception is guaranteed for a sufficiently long time after the transition state, soon there is a significant reduction of HPL to an acceptable value that is lesser than the alert limit of the application.

Dependability attributes depend on mutual relationship between protection level, alert limit and position error. The availability of position and HPL determination based on SBAS En-route/ NPA mode is $\mathrm{A}_{\mathrm{SIS}}=91.4 \%\left(\mathrm{~T}_{\mathrm{SIS}} \approx 8 \mathrm{~h}\right)$. These values have been determined for the entire track with consideration of 1 second GNSS update rate. However, applications can obtain information with the longer time interval $\Delta t$. Cases where the position is not determined or the alert limit is exceeded during the time interval $\Delta t$ occur only for the second application. Availability requirements with using GNSS SoL service are met, since the actual availability for all applications is higher than the required availability, see values of $A_{\min }$ in table 2 . Reliability of correct position determination and probabilities of different failure modes (safe, dangerous, detected, undetected) are also shown in table 2 . Minimum reliability $R_{\min }$ during the time interval $T_{S I S}$ is calculated on the basis of term $R_{\min }\left(T_{S I S}\right)=e^{-\lambda_{\max } T_{S I S}}$. Based on the numerical values in table 2, requirement for $R_{\min }$ is fulfilled for 1 second update time interval from GNSS receiver.
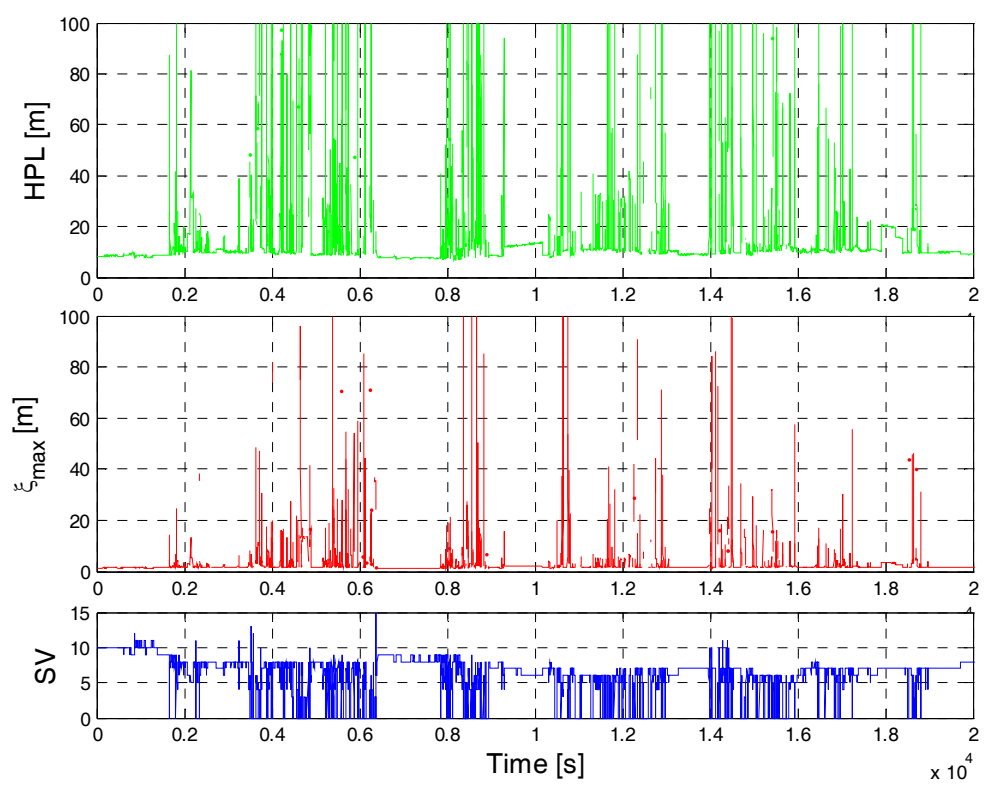

Figure 4: Time dependency of the measured data on the track. 
Table 2: Dependability attributes for railway applications.

\begin{tabular}{|c|c|c|c|}
\hline Application no. & 1 & 2 & 3 \\
\hline Availability of position and HPL on the track & \multicolumn{3}{|c|}{$\begin{array}{c}\mathrm{A}_{\mathrm{SIS}}=91.4 \%, \mathrm{~T}_{\mathrm{SIS}}=7.9 \\
h\end{array}$} \\
\hline $\mathrm{A}_{\min }-$ required & $90.6 \%$ & $91.1 \%$ & $91.1 \%$ \\
\hline $\mathrm{A}_{\min }-$ real & $100 \%$ & $96.7 \%$ & $100 \%$ \\
\hline $\mathrm{R}_{\min }\left(\mathrm{T}_{\mathrm{SIS}}\right)$ & $71.9 \%$ & $89.6 \%$ & $89.6 \%$ \\
\hline $\begin{array}{c}\text { Reliability (correct position determination, } \\
\text { i.e. no failure) } \\
\text { HPE } \leq \text { HPL } \leq \mathrm{HAL}, \mathrm{R}\left(\mathrm{T}_{\mathrm{SIS}}\right)=\end{array}$ & $95.7 \%$ & $96.2 \%$ & $95.7 \%$ \\
\hline $\begin{array}{c}\text { Safe undetected failure } \\
\mathrm{HPL}<\mathrm{HPE} \leq \mathrm{HAL}, \mathrm{PF}_{\mathrm{SU}}\left(\mathrm{T}_{\mathrm{SIS}}\right)=\end{array}$ & $0 \%$ & $0 \%$ & $0 \%$ \\
\hline $\begin{array}{c}\text { Dangerous undetected failure } \\
\mathrm{HPL} \leq \mathrm{HAL}<\mathrm{HPE}, \mathrm{PF}_{\mathrm{DU}}\left(\mathrm{T}_{\mathrm{SIS}}\right)=\end{array}$ & $0 \%$ & $0 \%$ & $0 \%$ \\
\hline $\begin{array}{c}\text { Safe detected failure, i.e. false alarm } \\
\mathrm{HPE} \leq \mathrm{HAL}<\mathrm{HPL}, \mathrm{PF}_{\mathrm{SD}}\left(\mathrm{T}_{\mathrm{SIS}}\right)=\end{array}$ & $3.9 \%$ & $3.4 \%$ & $3.9 \%$ \\
\hline $\begin{array}{c}\text { Dangerous detected failure, i.e. true alert } \\
\mathrm{HAL}<\mathrm{HPL}<\mathrm{HPE}, \mathrm{HAL}<\mathrm{HPE}<\mathrm{HPL}, \\
\operatorname{PF}_{\mathrm{DD}}\left(\mathrm{T}_{\mathrm{SIS}}\right)=\end{array}$ & $0.4 \%$ & $0.4 \%$ & $0.4 \%$ \\
\hline
\end{tabular}

\subsection{Significant limitation of satellite signal reception}

To analyze the usage of GNSS SoL service in adverse SIS reception conditions a lot of dynamic measurements were performed with a car in pre-selected critical areas. SIS reception was often insufficient and mentioned transition states have occurred very frequently in these areas.

Numerical values of dependability attributes from one specific experiment are expressed in table 3. The availability of positioning and protection level determination was only $47 \%$ during this measurement under very limited SIS reception conditions. Availability requirements are met, but reliability requirements are not met. This is caused by frequent occurrence of false alarms. The number of false alarms much exceeds that of true alerts. The reason for the occurrence of so many false alarms is that the protection levels in such adverse conditions of SIS reception do not reach the values smaller than the proposed alert limit of the application. No case of undetected failures was found.

Reliability could be only achieved by increasing of HAL to a tenfold value, which cannot be accepted for these applications. Viable solution for the usage of GNSS for railway applications in such critical environment can bring integration of GNSS with other sensors.

\subsection{Availability of GNSS navigation modes}

Improvement of availability of EGNOS NPA and PA navigation modes has been demonstrated in area of limited SIS reception by means of SIRF III technology. 
Table 3: Typical dependability attributes for the environment with adverse reception of SIS.

\begin{tabular}{|c|c|c|c|}
\hline Application no. & 1 & 2 & 3 \\
\hline $\begin{array}{c}\text { Availability of position and HPL on } \\
\text { the track }\end{array}$ & \multicolumn{3}{|c|}{$\mathrm{A}_{\mathrm{SIS}}=47 \%, \mathrm{~T}_{\mathrm{SIS}}=26$} \\
$\min$
\end{tabular}

Table 4: Availability of GNSS navigation modes.

\begin{tabular}{|c|c|c|c|}
\hline Satellites & $\begin{array}{c}\text { SIRF III } \\
\text { receiver }\end{array}$ & $\begin{array}{c}\text { PolaRx3: NPA } \\
\text { mode }\end{array}$ & $\begin{array}{c}\text { PolaRx3: PA / } \\
\text { autonomous }\end{array}$ \\
\hline 0 & $1 \%$ & $50 \%$ & $22 \%$ \\
\hline $1-3$ & $0 \%$ & $0 \%$ & $4 \%$ \\
\hline $4-6$ & $1 \%$ & $15 \%$ & $32 \%$ \\
\hline$>6$ & $98 \%$ & $35 \%$ & $42 \%$ \\
\hline
\end{tabular}

Table 4 shows that the receiver SIRF III receives mostly more than 6 satellites regardless of the adverse SIS reception conditions. It is obvious that the number of received satellites from SIRF III receiver is much higher in comparison with PolaRx 3 receivers. The first receiver PolaRx 3 was set in the NPA mode, while the second PolaRx3 receiver was configured in the PA/ autonomous mode. Table 4 also shows that availability of EGNOS SBAS navigation modes depends on the reception of SIS from geostationary satellites. The SIS reception of SBAS mode is available only from 3 geostationary satellites. For this reason, the NPA mode is unavailable for more than $28 \%$ of $\mathrm{PA} /$ autonomous mode. The future Galileo system will receive SBAS signal from all satellites that will also increase the availability of GNSS position determination and related quality measures.

\section{Conclusion}

This paper deals with the practical analysis of quantitative requirements for quality indicators of selected railway applications using GNSS SoL services. This analysis represents a part of the certification process of the GNSS system. Since the GNSS system is unable to meet demanding requirements for railway safety-related applications, the analysis was carried out only for non-safety related railway applications.

First of all, static measurements were performed and assumptions of the GNSS system behaviour under standard conditions of SIS reception were verified. Dynamic tests were then realized for subsequent analysis of vehicle 
position and protection level computation in adverse conditions of SIS reception in real railway environment. Experimental tests were carried out using GPS/ EGNOS receivers that meet requirements for the SoL applications.

In order to use GNSS system in railway safety-related applications, there is necessary to determine dependability attributes of the GNSS system and subsequently use them for design and verification of a safe train position locator consisting of several diverse sensors. Dependability assessment of the EGNOS system is the subject of our current research. It is particularly based on long-term experimental measurements and subsequent evaluation of measured data using the theory of random processes in time and frequency domains.

\section{Acknowledgement}

This work was supported by the Ministry of Transport of the Czech Republic under contract no. CG743-037-520.

\section{References}

[1] Mocek, H., Filip, A. \& Bažant, L., Galileo Safety-of-Life Service Utilization for Railway Non-Safety and Safety Critical Applications. STECH'09, Niigata, Japan, June 16-19, pp. 148-149, 2009.

[2] RTCA DO-229D. Minimum operational performance standards for GPS WAAS Airborne Equipment. RTCA, Inc., Washington, D.C., 2006.

[3] Filip, A., Beugin, J., Marais, J. \& Mocek, H., Safety Concept of Railway Signalling Based on Galileo Safety-of-Life Service. COMPRAIL 2008, Toledo, Spain, Sept 15-17, pp. 103-112, 2008.

[4] Galileo Integrity Concept. ESA document no. ESA-DEUI-NG-TN/01331, 2005. 